\title{
Why don't physicians enthusiastically support quality improvement programmes?
}

\section{P G Shekelle}

Resistance of physicians to clinical governance will continue until they can see how a real programme works operationally and a measurable leap in quality is achieved. In the absence of a role model, the opportunity exists for the NHS to fund primary care groups/trusts to develop a model that can be seen to work

$\mathrm{N}$ umerous studies over the past 20 years have documented the extent of quality problems with health care in most Western European and North American countries. Efforts to improve the quality of care have therefore taken on increasing urgency, and the Department of Health in the UK has now promoted clinical governance as a mechanism for quality assurance and improvement.

The paper by Campbell and colleagues in this issue of QSHC details the challenges for this policy in achieving its goal, in particular the role and concerns of physicians as to how clinical governance is going to be implemented. ${ }^{1}$ The resistance by physicians towards quality assurance and quality improvement efforts are, to my knowledge, common across all countries and health systems. This might seem paradoxical since, if one asked these physicians whether they wanted to deliver the best quality care possible to their patients, they would all say they did. Why, then, this apparent cognitive dissonance between the desire to deliver high quality care and resistance to organised efforts at quality assurance and improvement? I think there are at least four reasons.

The first is that physicians may not agree with the criteria by which quality is being measured. This concern has led to a great deal of expenditure of effort to improve the methods for case mix adjusting outcomes and the specification of processes so that clinicians feel the measures more accurately measure quality. Additionally, there is certainly a feeling on the part of some physicians that some measures of quality represent misplaced priorities, concentrating on those things that can be measured rather than on the things that are truly important for producing good patient outcomes. Fundamentally, however, I do not think that physicians disagree with most of the processes or most of the outcomes used or proposed for quality assurance and improvement. Even if we had perfect measures of quality, I think there would still be substantial physician resistance.

A second objection is that physicians view quality assurance and improvement programmes as an opportunity to blame them for anything bad that may or may not happen to the patient. This comes from the historical role of the physician as "the captain of the ship", responsible for all aspects of care the patient receives. In some countries, such as the USA, it also raises the fear of financial liability through malpractice litigation. These are very real concerns, and trying to shift the culture from one of blame to one of openness and learning from mistakes will be difficult. However, even if this could be accomplished, I do not believe that this will result in a substantially increased acceptance of quality assurance and improvement programmes on the part of physicians.

\section{"quality measurement and improvement will become as accepted and commonplace in health care as it is in virtually every other process in society"}

The third reason for physician resistance is that physicians believe they are being asked to participate in quality assurance and improvement programmes on top of all their other clinical and administrative responsibilities. All too often we see the situation where a quality assurance or improvement effort is mandated without any commitment of resources commensurate to the task at hand. Physicians who are already working long hours, dealing with sometimes complex management issues, simply do not see how they can be expected to put this additional time in on top of everything else. Physician resistance is not likely to lessen without adequate funding for quality improvement efforts.

However, even if we could get perfect agreement on the measures of quality, shift the culture from one of blame to one of learning from mistakes, and provide adequate resources for the tasks at hand, I think there will continue to be physician resistance until they can see in "nuts and bolts" detail how a real programme works operationally and makes a measurable quantum leap in quality. In other words, there are no role models here. And the reason there are no role models is that no one knows precisely how to do this. Here is where the opportunity exists for some fundholders such as the UK National Health Service to show real leadership. Since no one knows exactly how this should work, the NHS should invite primary care groups and trusts to submit proposals for systems of clinical governance designed to make major improvements in quality across multiple conditions, over a fixed period of time (say 3 years), to be measured by any of the plethora of validated technical quality and art of care measures now available. The carrot should be that the NHS will put a substantial amount of new resources into the primary care group/trust to help accomplish these goals. The stick should be that the primary care group/trust is held accountable for the results and that, unless certain measurable yardsticks are achieved, they will have to give back some or all of the money. Then let the best and most creative proposals surface, fund them, and see what happens.

From this process, a model or models will arise that can be shown to work. Once physicians see how it can be done, patients experience how quality has improved, and the NHS sees the vast improvements that can be had for the investment, then quality measurement and improvement will become as accepted and commonplace in health care as it is in virtually every other process in our societies, from manufacturing light bulbs to flying aeroplanes. Let's hope that day is not too far off.

Qual Saf Health Care 2002;11:6

Correspondence to: Dr P G Shekelle, West Los Angeles Veterans Affairs Medical Center, 11301 Wilshire Blvd, Los Angeles, CA 90073 , USA; shekelle@rand.org

\section{REFERENCE}

1 Campbell SM, Sheaff R, Sibbald B, et al. Implementing clinical governance in English primary care groups/trusts: reconciling quality improvement and quality assurance. Qual Saf Health Care 2002;11:9-14. 


\section{Barriers to incident reporting}

\section{J Firth-Cozens}

\section{Staff must be encouraged to report less serious incidents and near misses as well as more serious errors if lessons are to be learned and patient safety enhanced}

A key task in the enhancement of patient safety involves the ability to learn from error. ${ }^{1}$ The cultural change needed to achieve this requires staff to report the errors and near misses they commit or see others commit, and to use these data appropriately to change policy and practice. In the UK the National Patient Safety Agency has been set up as a body for the collection of errors so that the lessons-written large at a national rather than a local levelcan be appreciated more easily. However, this all depends upon errors being reported, and considerable research shows that this is very far from the case today.

The paper by Lawton and Parker in this issue of $Q S H C^{2}$ is important in showing what types of errors are likely to be reported and by whom-which is useful if we are to bring about change where reporting is not taking place. It shows that nurses and, to a lesser extent, midwives are much more likely to report incidents than doctors; that reporting is more common where protocols are in place and not adhered to; and that reporting is also more likely to occur when patients are harmed by the error.

These results begin to show the ways in which errors are perceived by different groups. They show the importance of protocols, which govern nurses far more than they do doctors, and that near misses are likely to go unreported, as are errors which occur when staff have to improvise outside protocols. This means that the lack of formal recognition of these types of errors may therefore fail to provide the opportunity for the development of new guidelines in this less charted territory. The importance of using all types of error to bring about safer care needs emphasising to staff, but this can only be done in an atmosphere of trust.

We may be heartened by the finding that all staff are more likely to report errors that cause actual harm to patients. This may be because they see these areas as the most important to address. However, it is also true that reporting of such incidents is much more difficult to avoid than is the reporting of less serious errors or near misses. Ironically, it is probably easier to learn from incidents which cause only minimal or no harm to patients, and are therefore less emotionally charged, than from serious events which may be surrounded by guilt, anguish, and fear. Staff need to be encouraged to report incidents which lead to less serious outcomes, but this will only happen in a non-punitive atmosphere that allows innovation and learning to flourish.

Qual Saf Health Care 2002;11:7

Correspondence to: Professor J Firth-Cozens, London Deanery, 20 Guilford Street, London WCIN IDZ, UK

i.firth-cozens@londondeanery.ac.uk

\section{REFERENCES}

1 Department of Health. An organisation with a memory London: Stationery Office, 2000.

2 Lawton R, Parker D. Barriers to incident reporting in a healthcare system. Qual Saf Health Care 2002;11:15-18.

\section{Telling patients the truth: a systems approach to disclosing adverse events}

\section{D Cantor}

The best way to improve disclosure of adverse events to patients and their families is to create a system for overseeing disclosure that is an integral part of the healthcare organisation's patient safety programme

$\mathrm{T}$ he best way to improve disclosure of adverse events (where the term "adverse event" means injury caused by the provision of health care rather than the patient's illness, whether or not the event resulted from a clearly identifiable error or mistake) to patients and their families is to create a system for overseeing disclosure that is an integral part of a healthcare organisation's patient safety programme. Cultural, legal, regulatory, and financial barriers prevent clinicians and healthcare organisations from disclosing adverse events, ${ }^{1-3}$ despite the ethical obligations of clinicians and healthcare organisations to do so. ${ }^{4-7}$ Applying a systematic continuous quality improvement model to disclosure of adverse events like the one proposed by Liang $^{8}$ in this issue of $Q S H C$ can help to overcome barriers to disclosure.

Effective disclosure of adverse events requires commitment to honesty and openness even when telling the truth may lead to loss of reputation, legal liability, or regulatory scrutiny. For clinicians the professional responsibilities of telling the truth and patient advocacy support disclosure of adverse events. ${ }^{67}$ From an organisational perspective, successful disclosure systems require a willingness to put the interests of patients and families first, and to maintain transparency, honesty, and trust. Patient safety systems only work when there is an atmosphere that permits and supports open exchange of information, whether it is through reporting systems, disclosure, or investigation of the root causes of adverse events. ${ }^{9}$ Disclosure of adverse events can enhance patient safety by reinforcing the values important to a culture of safety-honesty, respect, and transparency.

Disclosure partly depends on whether other parts of the patient safety system are working. It cannot occur unless adverse events are identified in a timely manner and brought to the attention of the disclosure programme. Without investigation of adverse events, it can be difficult to know what to disclose.

At the heart of an effective disclosure system are clear policies that provide 
guidance on whether and what to disclose, who should disclose, and how disclosure should occur. Determining whether to disclose is complicated by the many different types of adverse events and the differing amounts of harm they cause. Patients want to be informed of even minor adverse events, ${ }^{10}$ but others argue that patients need to be informed of adverse events only when these events result in harm to the patient. ${ }^{11}$ Liang suggests that near miss errors should be disclosed "to provide opportunities for systems learning that may be important for potentially serious adverse events" ${ }^{8}$

\section{"At the heart of an effective disclosure system are clear policies that provide guidance on whether and what to disclose, who should disclose, and how disclosure should occur."}

Given the practical limits of time and availability of staff to make disclosures and the relatively low likelihood that disclosures of near misses would elicit information that could not be obtained through a near miss reporting system, it makes sense to focus disclosure on adverse events that cause harm. The standard of the Joint Commission on Accreditation of Healthcare Organizations (JCAHO) - the body that accredits US hospitals-that went into effect in June 2001 requires hospitals to encourage disclosure of "unanticipated outcomes" and therefore supports disclosure of events that have actually harmed patients. When is the harm caused too trivial to disclose? Has the patient who received the wrong medication but only had a temporary drop in blood pressure suffered enough harm to have the event disclosed? Systematising the process of disclosure will enable clinicians, administrators, and attorneys responsible for managing adverse events to build a consensus about whether disclosure is necessary.

Determining who should disclose an adverse event is also controversial and systems have chosen different approaches. The system in place at the Kentucky Veterans Affairs Medical Center, Lexington $^{13}$ and the model proposed by Liang ${ }^{8}$ remove the clinician from the disclosure process and instead place responsibility and the process of disclosure with organisational leaders and a risk management team. In contrast, the University of PittsburghPresbyterian Medical Center policy places responsibility for notifying the patient or family about the adverse event on the attending physician. ${ }^{14}$ The interpretation provided by the JCAHO standard requires the "responsible licensed independent practitioner or his or her designee [to] clearly explain the outcome of any treatments or procedures., ${ }^{12}$

Deciding who should disclose reflects the underlying philosophy of the organisation. If safety is seen primarily as an organisational/systems issue, then the organisation should bear complete responsibility for the disclosure process. If clinicians are seen as primarily responsible for assuring safety, then it makes sense for clinicians to take the lead in disclosing. In reality, responsibility for patient safety is shared by clinicians and the organisation/system within which they work. The best system encourages involvement of clinicians within an organisational process that is supportive of disclosure. Even if primary responsibility rests with a disclosure team, clinicians should be given the opportunity to participate in the process of disclosure. If either clinician disclosure or organisation led disclosure is the default policy, the decision as to who should lead the disclosure process should be made on a case by case basis that takes account of the nature of the adverse event, the relationship between the patient and the clinician involved, and the skill of the clinician in effectively disclosing the adverse event.

Once the decision has been made to disclose and responsibility for disclosure has been determined, attention must be paid to the manner in which disclosure occurs. Policies and procedures should incorporate Buckman's principles for breaking bad news. ${ }^{15}$ This approach is useful in communicating bad news to patients and can be taught to clinicians and other participants who need to improve their communication skills.

Creating a system for managing disclosure of adverse events to patients and/or family members is critical to improving patient safety. As organisations struggle to implement patient safety techniques and change their culture to one of openness and honesty, disclosure of adverse events should be seen as an opportunity for organisations to demonstrate their commitment to putting the needs of patients first. There is no single solution that will work in every organisation, but those organisations that choose a systematic approach are most likely to succeed.

Qual Saf Health Care 2002;11:7-8

Correspondence to: Dr M D Cantor, VHA

National Center for Ethics (10AE), VACO, 810

Vermont Ave, N W Washington, DC 20420,

USA; Michael.Cantor@hq.med.va.gov

The views expressed in this article do not necessarily represent the views of the US Department of Veterans Affairs or the official policy of the Veterans Health Administration.

\section{REFERENCES}

1 Kohn LT, Corrigan JM, Donaldson, MS, eds. Committee on Quality of Health Care In America, Institute of Medicine. To err is human: building a safer health system. Washington, DC: National Academy Press, 2000.

2 Wu AW, Cavanaugh TA, McPhee SJ, et al. To tell the truth: ethical and practical issues in disclosing medical mistakes to patients. J Gen Intern Med 1997; 12:770-5.

3 Barach P, Small SD. Reporting and preventing medical mishaps: lessons from non-medical near miss reporting systems. $B M$ 2000;320:759-63.

4 Smith ML, Forster HP. Morally managing medical mistakes. Camb Q Healthc Ethics 2000;9:38-53.

5 Thurman AE. Institutional responses to medical mistakes: ethical and legal perspectives. Kennedy Inst Ethics J 2001;11:147-56.

6 American Medical Association, Council on Ethical and Judicial Affairs. Code of medical ethics: current opinions with annotations. Chicago, IL: American Medical Association, 1997: §8.12.25

7 DeVita MA. Honestly, do we need a policy on truth? Kennedy Inst Ethics J 2001;11:157-64.

8 Liang BA. Honesty and transparency with patients: a system for medical error disclosure. Qual Saf Health Care 2002:11:64-8.

9 Billings CE. Some hopes and concerns regarding medical event reporting systems: lessons from the NASA aviation safety reporting system (ASRS). Arch Pathol Lab Med 1998;121:214-5.

10 Witman AB, Park DM, Hardin SB. How do patients want physicians to handle mistakes? A survey of internal medicine patients in an academic setting. Arch Intern Med 1996; 156:2565-9.

11 Lo B. Resolving ethical dilemmas: a guide for clinicians. Baltimore: Williams \& Wilkins, 1995.

12 Joint Commission on Accreditation of Healthcare Organizations. Comprehensive accreditation manual for hospitals. Standard RI 1.2.2. Chicago, IL: Joint Commission on Accreditation of Health Care Organizations, 2001.

13 Kraman SS, Hamm G. Risk management: extreme honesty may be the best policy. Ann Intern Med 1999;131:963-7.

14 UPMC Presbyterian Policy and Procedure Manual. Guidelines for disclosure and discussion of conditions and events with patients, families and guardians. Kennedy Ethics Inst J 2001;11:165-8.

15 Buckman R, Kason Y. How to break bad news: a guide for health care professionals. Baltimore, MD: Johns Hopkins University Press, 1992. 